\title{
Applying a System for Measuring Dynamic Knowledge: Reconsidering the Spiral Model
}

\author{
Mark E Nissen \\ US Naval Postgraduate School \\ MNissen@nps.edu
}

\begin{abstract}
Knowledge is key to competitive advantage, but it is inherently invisible, intangible and resistant to quantification, particularly when in dynamic motion. Recent research builds upon emerging knowledge measurement techniques and well-established knowledge flow theory to develop a system for measuring dynamic knowledge in the organization. Results from application to archetypical organization processes are highly consistent with much theory. However, they also lead us to question some longstanding theoretic concepts and principles. In this article, we reconsider the well-known Spiral Model through dynamic knowledge measurement.
\end{abstract}

\section{Introduction}

Knowledge is key to competitive advantage [3,7,22]: Knowledge enables effective action; effective action drives superior performance; and superior performance supports competitive advantage [13]. Indeed, some scholars argue that knowledge represents the only sustainable source of competitive advantage [5].

However, knowledge does not represent a single, monolithic concept: different kinds of knowledge (e.g., tacit, explicit, individual, group, created, applied) have qualitatively different properties and behaviors, and hence affect action, performance and competitive advantage differently [11]. Neither can knowledge remain static in support of competitive advantage: it must move or flow rapidly and reliably from where and when it is to where and when it is needed in the organization.

This places particular importance on understanding the dynamics of knowledge as it flows, but unfortunately, knowledge is inherently intangible, invisible and resistant to quantification [1], particularly when in dynamic motion. This makes it a considerable and persistent challenge to understand, visualize and measure.
Recent research builds upon emerging knowledge measurement techniques and well-established knowledge flow theory to develop a system for measuring dynamic knowledge in the organization [14]. Results from application to archetypical organization processes are highly consistent with much theory. For instance, measured differences between the dynamics of tacit and explicit knowledge flows mirror theoretic predictions. However, they also lead us to question some longstanding theoretic concepts and principles. For instance, the concept knowledge spiral [15] exhibits difficulties when instantiated via dynamic knowledge measurements.

In this article, we review a system for measuring dynamic knowledge in the organization, and we illustrate its theoretic consistency through measurement of knowledge flow archetypes from the literature. We then apply dynamic knowledge measurements to instantiate the knowledge spiral. Such application and instantiation lead us to reconsider the well-known Spiral Model and catalyze continued research along these lines.

\section{Background}

After casting a wide metaphoric net in terms of relevant literatures to review (e.g., Economics, Education, Information Theory, Knowledge Management) for background, insight and inspiration [14], the research noted above draws judiciously and analogically from our understanding of dynamic physical systems to conceptualize a set of equations for measuring dynamic knowledge.

As a fundamental cognitive process [17], employed by adults [23] and children [24] alike, analogic reasoning represents a notably powerful learning and communication approach that spans many domains, including Design [2], Organization [26], Physics [18], Strategy [6], Supply Chain [9], and many others. Analogies can promote creativity, in both people and computers [8], and they can facilitate thinking in domains with negligible precedent, such as Outer Space Law [16]. 
Nonetheless, it is important to emphasize that we recognize the limitations of analogic reasoning: In no way are we asserting that the dynamics of knowledge follow or mirror the dynamics of physical systems precisely. Every analogy breaks down when stretched too far, and even some of the most basic physical concepts may have little meaning in terms of dynamic knowledge. Notwithstanding such limitations, however, we gain insight from the deep understanding and mathematic representation of dynamic physical systems, which are adapted here to enable the measurement of dynamic knowledge.

\section{Knowledge Measurement System}

In this section we outline a simple set of dynamic physical equations, which we analogize in turn to conceptualize a comparable set of dynamic knowledge equations.

\subsection{Dynamic physical equations}

To recapitulate the approach, which is described in detail through the research noted above [14], a simple physical system is represented mathematically through the basic Newtonian equations summarized in Table 1. Such equations can be found in any introductory Physics textbook, yet they enable quantitative measurement, analysis, prediction and simulation of dynamic physical systems. Here we interrelate force (mass $\mathrm{x}$ acceleration; expressed in Newtons), work (force $\mathrm{x}$ distance; expressed in Joules) and power (work / time; expressed in Watts).

Table 1 Physical System Equations

\begin{tabular}{|l|l|l|}
\hline Construct & \multicolumn{1}{|c|}{ Description } & Equation \\
\hline Force (F) & $\begin{array}{l}\text { Effort required to } \\
\text { accelerate mass }\end{array}$ & (1) F = m x a \\
\hline Work (W) & $\begin{array}{l}\text { Force applied } \\
\text { through distance }\end{array}$ & (2) W = F x d \\
\hline Power (P) & $\begin{array}{l}\text { Work done per unit } \\
\text { time }\end{array}$ & (3) P = W / t \\
\hline
\end{tabular}

We also note (beyond the table) how work and energy are exchangeable and expressed in the same units (Joules): energy is required to perform work, and work performance involves the expenditure of energy. We leverage such exchangeability below through analogic reasoning for knowledge systems.

We note further how friction affects many physical systems by impeding acceleration. An ordinary shopping cart, for instance, requires greater effort (i.e., more force) to push down a store aisle with a rough floor than a smooth one: the greater friction associated with the rough floor impedes acceleration of the cart, hence it requires more force to push.

Considering friction in support of our analogic reasoning, a simple, linear, negative relationship between force-including that required to overcome friction $\left(\mathrm{F}_{\mathrm{Fr}}\right)$ - and floor smoothness (fs) is delineated in Figure 1. Here force can be measured in Newtons, and smoothness is expressed on a $[0,1]$ continuum between rough $(\mathrm{fs}=0)$ and smooth $(\mathrm{fs}=1)$ endpoints.

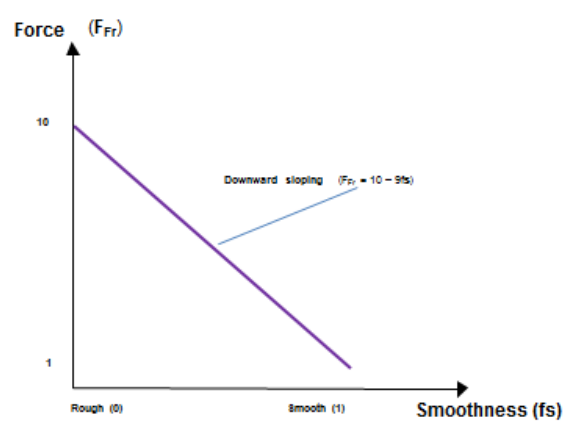

Figure 1 Force and Smoothness

Specifically as depicted in the figure, a rough floor is characterized here as requiring ten times the force to push a shopping cart as that needed on a smooth floor $\left(\mathrm{F}_{\mathrm{Fr}}=10\right.$ - 9fs). This downward sloping relationship between force and smoothness is representative, with specific slopes, intercepts and functions highly likely to differ across various carts, stores, aisles and floors. Nonetheless, the relationship makes intuitive sense and is consistent with many physical observations and measurements.

\subsection{Dynamic knowledge equations}

As summarized in Table 2, we outline an analogic system of equations for measuring dynamic knowledge. To reiterate from above, none of these analogic constructs or relationships is precise or perfect, yet they compose a simple, novel and insightful system for measuring dynamic knowledge.

Briefly, knowledge force (KF) is analogous to physical force and represents the effort required to accelerate knowledge in an organization. From Knowledge Flow Theory (KFT; see $[4,7,12,15,20,22])$, it is expressed as a function of the knowledge chunks (C) [21] being accelerated and the explicitness (E) of such knowledge. In this conceptualization, one chunk of knowledge can enable the performance of one atomic action in the 
organization. Explicitness derives from Nonaka's [15] epistemological dimension and represents the degree to which a knowledge chunk has been articulated in explicit form. The greater the number of chunks being accelerated (analogous to physical mass), and the more tacit the corresponding knowledge (analogous to physical friction), the greater the K-Force required. Notice also the $\mathbf{0}$ vector representing a number of other, unspecified factors (e.g., communication skill, motivation, stress, organization climate, IT support), which are likely to play a role, but which have yet to be integrated explicitly or analogically.

Table 2 Analogic Knowledge System

\begin{tabular}{|l|l|l|}
\hline Construct & \multicolumn{1}{|c|}{ Description } & Analogy \\
\hline $\begin{array}{l}\text { K-Force } \\
(\mathrm{KF})\end{array}$ & $\begin{array}{l}\text { Effort required to } \\
\text { accelerate knowledge }\end{array}$ & $\mathrm{f}(\mathrm{C}, \mathrm{E}, \mathbf{o})$ \\
\hline $\begin{array}{l}\text { K-Work } \\
(\mathrm{KW})\end{array}$ & $\begin{array}{l}\text { K-Force applied } \\
\text { through reach }\end{array}$ & KF x R \\
\hline $\begin{array}{l}\text { K-Power } \\
(\mathrm{KP})\end{array}$ & $\begin{array}{l}\text { K-Work done per unit } \\
\text { flow time }\end{array}$ & KW / FT \\
\hline
\end{tabular}

Reach (R) derives from Nonaka's [15] ontological dimension and represents the number of people able to access and utilize the knowledge chunks from above (analogous to physical distance). Reach combines with K-Force to specify knowledge work (KW) accomplished in the organization (analogous to physical work). Analogous to the exchange between and common units of work and energy in physical systems, we also conceptualize a correspondence between knowledge work and knowledge energy (KE): K-Energy is required to perform K-Work, and $\mathrm{K}$-Work performance involves the expenditure of $\mathrm{K}$ Energy.

In turn, flow time (FT) represents the time required for such knowledge chunks to flow from one person (e.g., an expert), group (e.g., a sales team), place (e.g., West Coast office) or time (e.g., night shift) to another. As a time measure, it combines with KW to specify knowledge power (KP), which represents the knowledge work accomplished (and knowledge energy expended) per unit time (analogous to physical power).

Continuing to draw analogically from the dynamics of physical systems, and considering friction, which impedes acceleration, a simple, linear, negative relationship between knowledge force (KF) and explicitness (E) is delineated in Figure 2. Consistent with KFT, this relationship indicates that tacit knowledge, which is notably "sticky" [25] and difficult to move through the organization, requires more effort (i.e., greater $\mathrm{KF}$ ) to accelerate than its explicit counterpart.
Alternatively, tacit knowledge, in the context of which Polanyi [19] explains that we know more than we can tell, can enable knowledge work at higher performance levels than explicit. Reading a book (i.e., explicit knowledge) about how to fly an airplane, for instance, is not the same as direct experience (i.e., tacit knowledge) flying airplanes and is unlikely to enable performance at the same level.

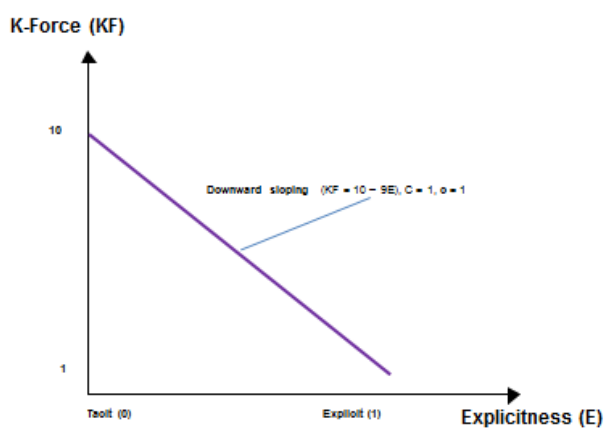

\section{Figure 2 Knowledge Force and Explicitness}

Specifically as depicted in the figure, a chunk of tacit knowledge is characterized here as requiring (somewhat arbitrarily but analogously) ten times (10x) the K-Force needed to get a chunk of explicit knowledge flowing (KF = $10-9 \mathrm{E})$. Space prohibits a long discussion of sensitivity analysis, but results are highly robust to differences in slope (e.g., 2x, 100x), linearity (e.g., $\mathrm{x}^{2}, \mathrm{x}^{1 / 2}$ ) and other factors.

Indeed, this downward sloping relationship between K-force and explicitness is representative, with specific slopes, intercepts and functions highly likely to differ across various organizations, people, processes and kinds of knowledge. Nonetheless, the relationship makes intuitive sense and is analogous to physical friction.

Further, we can use this representative relationship to specify the set of dynamic knowledge equations summarized in Table 3. In Equation (4) we specify K-Force as a multiplicative function of knowledge chunks (C), explicitness (10 - 9E), and vector of unspecified other factors (o). We refer to units of K-Force as "Nonakas" $(\mathrm{N})$, acknowledging the seminal knowledge flow research done by Nonaka [15].

Table 3 Knowledge System Equations

\begin{tabular}{|l|l|}
\hline Construct & \multicolumn{1}{c|}{ Equation } \\
\hline K-Force & (4) KF = C x (10 - 9E) x o \\
\hline K-Work & (5) KW = KF x R (= KE) \\
\hline K-Power & (6) KP = KW / FT \\
\hline
\end{tabular}


K-Work (and K-Energy) then follows in Equation (5) as the product of K-Force and reach (R). We refer to units of K-Work (and K-Energy) as "Polanyis” (P), for the keen insight into tacit knowledge provided by Polanyi [19]. K-Power is specified in turn through Equation (6) by dividing K-Work by flow time. We refer to K-Power as "Bacons” (B), acknowledging Sir Francis Bacon, to whom many scholars attribute the aphorism, "knowledge is power."

To reiterate from above, this analogical reasoning is not strict, and we recognize its limitations. Nonetheless, we gain insight from the deep understanding and mathematic representation of dynamic physical systems, which are adapted here to address the measurement of dynamic knowledge, and even this simple set of equations enables us to begin measuring knowledge as it flows through the organization. This represents a substantial step forward in terms of knowledge measurement.

\section{Archetypical Application}

In this section we apply the set of knowledge equations to knowledge flow archetypes from the literature. First we draw from recent research and outline a multidimensional approach to visualizing dynamic knowledge, which utilizes many of the measurement constructs described above. Then we apply the corresponding measurement system directly.

\subsection{Dynamic knowledge visualization}

To briefly outline this multidimensional visualization approach, which is described in detail through the research noted above [14], we refer to Figure 3. The vertical axis represents explicitness, which is one of the knowledge measurement constructs from above and derives from Nonaka [15]. The horizontal axis represents reach, which is another of the knowledge measurement constructs from above and derives from Nonaka [15] also. The third axis represents life cycle, which is helpful for visualization and used to extend Nonaka's model [10]. Life cycle pertains to what is being done with knowledge (e.g., create, share, apply).

Flow time is not delineated via separate axis, but it is another of the knowledge measurement constructs from above and used to extend Nonaka's model further. Within the context of this multidimensional visualization scheme, flow time represents the time required for knowledge to flow between any two coordinate points in the space (e.g.,
Points A and B in the figure). When knowledge flows quickly through an organization (i.e., when flow time is short), for instance, we delineate the corresponding flow with a relatively thin vector arrow, whereas a comparatively thick one is used when knowledge flows slowly. Our expectations from KFT are that tacit knowledge, which is notably "sticky" and difficult to move through the organization, will flow more slowly than its explicit counterpart. Hence tacit flows would generally be represented by relatively thick arrows, whereas comparatively thin ones reflect explicit flows better.

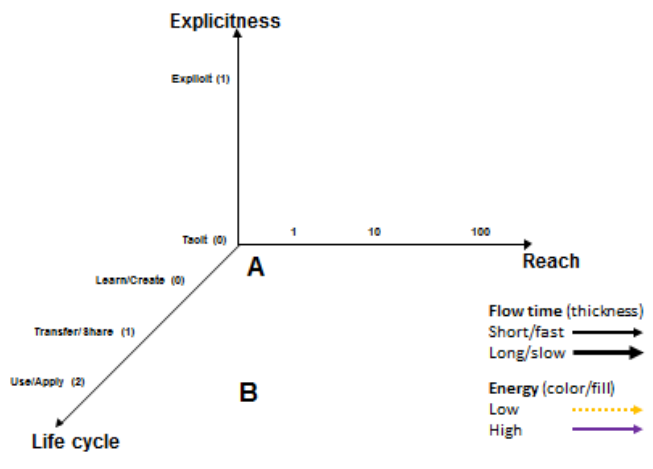

Figure 3 Knowledge Visualization Space

Finally, we also utilize different arrows to delineate knowledge energy, which is noted above with correspondence to the measurement construct knowledge work, and which represents the efficacy or performance level of actions enabled by knowledge as it flows through the organization. Higher energy knowledge flows (e.g., that enable higher levels of knowledge work performance) are delineated with solid (purple) vector arrows, for instance, whereas dotted (orange) arrows are used for lower energy knowledge. Our expectations from KFT are that tacit knowledge, which can enable higher performance levels, will flow with more energy than its explicit counterpart. Hence tacit flows would generally be represented by solid (purple) arrows, whereas dotted (orange) ones reflect explicit flows better. In theory, flow time and knowledge energy represent orthogonal dimensions, but in practice, they may covary.

In terms of measurement, explicitness can be represented as a continuous dimension, with tacit and explicit endpoints on a ratio scale (e.g., [0, 1]). This implies that various combinations of tacit and explicit streams may comprise some knowledge flows. Such conceptualization as a continuous dimension also serves to extend much prior research (e.g., [15]), which views tacit and explicit knowledge more as a categorical contrast than a continuum. Reach can be 
measured along an integer scale (e.g., 1, 10, 100), enumerating the number of people who can utilize knowledge. Life cycle represents an iterative sequence of activities, with a somewhat arbitrary ordinal scale (e.g., $0,1,2)$ referring to what is being done with knowledge. Flow time can be measured along a ratio scale using a stopwatch, calendar, employee timecard, or like instrument. As noted above, K-Energy (and K-Work) is calculated as the product of K-Force and reach.

Together, this multidimensional framework enables the visualization of dynamic knowledge and is very general. Theoretically, any dynamic flow of knowledge can be characterized in terms of these dimensions and delineated in this space, and in theory, knowledge can flow via an infinite number of different paths between any two points.

Consider, for example, Points $\mathrm{A}$ and $\mathrm{B}$ in the figure. Say that an individual worker in the organization discovers some new and useful knowledge (Point A), and management is interested in having all ten people in a group learn and apply such knowledge (Point B). There are clearly many different organization sharing processes available to enable this new knowledge to flow between such individual and group members, hence equally many corresponding knowledge flow paths through the multidimensional space are possible too.

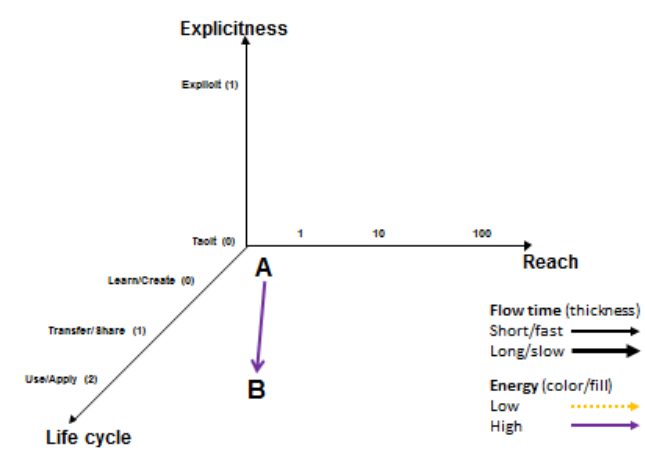

Figure 4 Ideal Knowledge Flow

The thin, solid (purple) vector arrow connecting Points A and B in Figure 4, for instance, represents an ideal archetypical knowledge flow path: high energy tacit knowledge, which can enable knowledge work at high performance levels in the organization, is depicted as flowing quickly and directly, in a short, straight line, from A to B. A straight line delineates the shortest distance between two points in space, and this vector depicts the fastest, highest energy knowledge flow between Points $\mathrm{A}$ and $\mathrm{B}$, hence its classification as "ideal."
In practice, however, the number of feasible knowledge flow paths is likely to be limited. The fast, direct, high energy flow vector delineated in Figure 4, for instance, may be ideal, but it's doubtful that any organization is capable of accomplishing such flow. Indeed, as noted above, tacit knowledge is notably "sticky" and tends to flow slowly, hence the flow vector arrow should be thicker, and this arrow should also pass through an intermediate knowledge sharing step before it can be applied directly by the group members (e.g., members need to learn the knowledge via sharing before being able to apply it). Identifying feasible knowledge flow paths, and selecting the best ones for a given organization context and situation, represents an important management capability.

Consider, as a more feasible example, the archetypical knowledge flow labeled "Explicit Path" in Figure 5. Say that the individual worker (Point A) expends time and energy to articulate his or her knowledge in explicit form (e.g., written instructions, graphic depictions, mathematic formulae and calculations, solved examples). This is represented by Point $\mathrm{M}$ in the figure. Then this individual could encode such explicit knowledge digitally within a computer network (e.g., via email attachment, website resource, document repository), which could be shared very quickly with all ten coworkers, wherever in the world they happen to be located. This is represented by Point $\mathrm{N}$ in the figure.

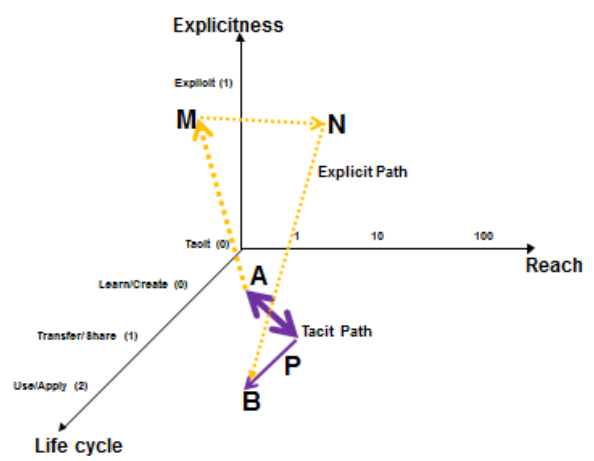

Figure 5 Knowledge Flow Archetypes

After sharing as such, each of the coworkers could apply the knowledge directly to his or her work activities (Point B). This organization process and corresponding knowledge flow path are illustrated by light (orange) dotted vector arrows in the figure to represent the explicit nature of the dynamic knowledge. The first segment (i.e., A-M) is delineated with a relatively thick vector to indicate that the process of articulating tacit knowledge into 
explicit form can be time consuming, particularly when compared to the other segments corresponding to explicit knowledge sharing (i.e., $\mathrm{M}-\mathrm{N}$ ) and application (i.e., N-B). By using a stopwatch, calendar, employee timecard, or like instrument, researchers or managers could measure the time required for this knowledge to flow from $\mathrm{A}$ to $\mathrm{B}$, and hence obtain a measured value for flow time.

Consider, as a contrasting feasible example, the archetypical knowledge flow labeled “Tacit Path” in Figure 5. Say that the individual worker interacts interpersonally with the group members, working closely with these people, soliciting and answering their questions, observing and correcting the coworkers as they practice, and both mentoring and coaching them until everyone in the group has learned the knowledge. This is represented by Point $\mathrm{P}$ in the figure.

With such learning accomplished effectively, all ten coworkers would be able to apply the knowledge directly to their work activities (Point B). This Tacit Path differs greatly from the Explicit Path above, and the corresponding knowledge flow is illustrated by dark (purple) solid vector arrows in the figure to represent the tacit nature of the dynamic knowledge.

The first segment (i.e., A-P) is delineated with a relatively thick arrow to indicate that the process of sharing tacit knowledge can be especially time consuming, particularly when compared to the other segment corresponding to tacit knowledge application (i.e., P-B). This first segment is delineated with a double headed arrow also to indicate that knowledge sharing goes both ways: the individual worker (Point A) is learning (e.g., group norms) from the other members as they interact interpersonally, and the coworkers are learning (esp. the new knowledge) from this individual.

As above, researchers or managers could use the same stopwatch, calendar, employee timecard, or like instrument to measure the time required for knowledge to flow from $\mathrm{A}$ to $\mathrm{B}$, and hence obtain a measured value for flow time along this alternate, tacit path. Since these two, contrasting, archetypical knowledge flow paths are very different, one would expect for the corresponding flow times and energy levels to differ accordingly.

\subsection{Dynamic knowledge measurement}

For any of the flow paths delineated above, let's say that the new knowledge involves 100 chunks. This would imply that such new knowledge enables approximately 100 novel actions to be performed. Let's say further that everyone in this organization is diligent about maintaining detailed records of how they spend their time in the office, hence researchers or managers can obtain flow time measurements from the workers' timecards. Here we illustrate how to obtain measurements for the three knowledge flow archetypes delineated and described above. This begins with the Ideal Path, which, although infeasible in practice, represents a noteworthy theoretic flow pattern for reference. Measurements are summarized in Table 4.

Table 4 Ideal Path Measurement

\begin{tabular}{|r|c|c|c|c|c|c|}
\hline Flow & E & KF & R & KW & FT & KP \\
\hline A-B & 0.0 & 1.00 & 10 & 10.00 & 0.1 & 100.00 \\
\hline
\end{tabular}

Walking across columns in the table, for the 100 chunks moving through this flow (A-B), one can see explicitness (0.0) in Column 2 denotes purely tacit knowledge. Using Equation (4), this results in KForce of $1000 \mathrm{~N}$ (KF, KW and FT are expressed in thousands in the table), and with reach (10) for the whole group of coworkers, Equation (5) indicates KWork (and K-Energy) of 10,000 P. The workers' time records indicate that only a couple minutes (100 seconds) of flow time are required for application. This results in K-Power of $100 \mathrm{~B}$. We gain perspective below through comparison with the other knowledge flow archetypes.

Table 5 Explicit Path Measurement

\begin{tabular}{|r|c|c|r|r|r|r|}
\hline Flow & E & KF & R & KW & FT & KP \\
\hline A-M & 0.5 & 0.55 & 1 & 0.55 & 15.0 & \\
\hline M-N & 1.0 & 0.10 & 10 & 1.00 & 0.1 & \\
\hline N-B & 1.0 & 0.10 & 10 & 1.00 & 1.0 & \\
\hline Sum & & & & 2.55 & 16.1 & 0.16 \\
\hline
\end{tabular}

Measurements corresponding to the Explicit Path are summarized in Table 5. Notice that we divide the measurements into three parts corresponding to each of the flow vectors noted above. For the same 100 chunks moving through the first flow (A-M), one can see explicitness is listed as a fractional value (0.5) in Column 2. This denotes that knowledge associated with the flow begins as tacit $(\mathrm{E}=0)$ and ends as explicit $(E=1)$, as the individual worker articulates tacit knowledge into explicit form. Using the same equations noted above, this results in K-Force of 550 $\mathrm{N}$, and with unitary reach (i.e., the individual), KWork of 550 P. The worker's time records indicate that just over four hours are invested in articulating the knowledge in explicit form and making it available on the computer network, which corresponds to $15,000 \mathrm{~s}$ flow time.

Calculations for the other two flow vectors (M-N, N-B) involve the same 100 knowledge chunks and follow the same logic and procedure. Notice that 
knowledge is purely explicit for these latter flow segments and that both involve the same reach (10) across the group. In the first (M-N), explicit knowledge flows very quickly $(100 \mathrm{~s})$ and simultaneously to all ten people via computer network. In the second (N-B), all ten coworkers apply such explicit knowledge directly and in parallel, through actions requiring nearly 17 minutes (1000 s) to complete.

K-Work (and K-Energy) is nearly double for these purely explicit flows because of the greater reach (10 vs. 1). Summing K-Work (2550 P) and flow time $(16,100 \mathrm{~s})$ for the process as a whole, (average) K-Power of $0.16 \mathrm{~B}$ obtains, which provides a numeric measurement and basis for comparison with the Ideal Path from above. Clearly knowledge flowing through the Explicit Path takes considerably longer and accomplishes much less K-Work (at a miniscule relative K-Power level than that moving through the Ideal Path, for instance.

Measurements corresponding to the Tacit Path are summarized in Table 6. They involve the same 100 knowledge chunks and follow the same logic and procedure described above. Notice that knowledge for both flow vectors comprising this latter process is purely tacit $(E=0)$. This reflects the kind of interpersonal, iterative, experiential interaction that is associated widely with tacit knowledge sharing. As such, and as above, the system of equations is used to obtain the measurement values in this table for the tacit knowledge flow path, and flow time from coworkers' time records is included.

Table 6 Tacit Path Measurement

\begin{tabular}{|l|c|c|c|c|r|c|}
\hline Flow & E & KF & R & KW & \multicolumn{1}{|c|}{ FT } & KP \\
\hline A-P & 0.0 & 1.0 & 10 & 10.0 & 55.0 & \\
\hline P-B & 0.0 & 1.0 & 10 & 10.0 & 0.1 & \\
\hline Sum & & & & 20.0 & 55.1 & 0.36 \\
\hline
\end{tabular}

Notice further that a relatively long time (i.e., roughly 15 hours; FT $=55,000 \mathrm{~s}$ ) is required for this tacit knowledge to flow (A-P). This is consistent with the "sticky" nature of tacit knowledge. Alternatively, once learned, application of such tacit knowledge is comparatively very quick (i.e., FT $=100 \mathrm{~s}$ ) for the group of coworkers (P-B). As with the Explicit Path above, knowledge flowing through the Tacit Path takes considerably longer than that moving through the Ideal Path, but it accomplishes double the KWork. Nonetheless, the K-Power $(0.36)$ is also miniscule relative to the Ideal Path (100.00).

Other comparisons across the three archetypical knowledge flow paths are noteworthy, and some comparative measurements are summarized in Table 7. For one, the Ideal Path reflects enormous K-Power
(100 B), illustrating the accomplishment of much KWork (10 P) in very little time (100 s).

Looking now at the Explicit and Tacit Path archetypes, the tacit flow accomplishes nearly eight times the knowledge work (and expends comparably greater K-Energy) as its explicit counterpart (20,000 vs. $2550 \mathrm{P}$ ), but it takes more than three times as long to accomplish such work (55,100 vs. 16,100 s). Which of these two, feasible archetypes is "best"? The K-Power metric reveals that the tacit path completes the knowledge flow at over double the power level (0.36 vs. 0.16 B) of its explicit counterpart. This indicates that the additional knowledge work accomplished through the former process more than makes up for the greater amount of time required for the knowledge to flow. These measurements are highly consistent with KFT.

Table 7 Knowledge Flow Path Comparison

\begin{tabular}{|l|r|r|r|l|}
\hline Path & \multicolumn{1}{|c|}{ KW } & \multicolumn{1}{c|}{ FT } & KP & Comment \\
\hline Ideal & 10.00 & 0.1 & 100.00 & $\begin{array}{l}\text { High power } \\
\text { Infeasible }\end{array}$ \\
\hline Explicit & 2.55 & 16.1 & 0.16 & $\begin{array}{l}\text { Less work } \\
\text { Less time }\end{array}$ \\
\hline Tacit & 20.00 & 55.1 & 0.36 & $\begin{array}{l}\text { More work } \\
\text { More time }\end{array}$ \\
\hline $\begin{array}{l}\text { T:E } \\
\text { Ratio }\end{array}$ & 7.8 & 3.4 & 2.3 & $\begin{array}{l}\text { "Best”? } \\
\text { K-Power }\end{array}$ \\
\hline
\end{tabular}

\section{Spiral Model Application}

Here we review knowledge spiral basics, and as with the archetypical flow paths delineated above, we instantiate the associated theoretic model through both multidimensional knowledge visualization and dynamic measurement.

Briefly, the knowledge spiral [15] conceptualizes organization knowledge flowing through iterative conversions (i.e., socialization, externalization, combination, internalization). Each conversion involves tacit andlor explicit knowledge.

Socialization is a tacit-to-tacit flow, as an individual learns experientially from others, for instance. This is similar in many respects to how the Tacit Path from above begins, where knowledge is shared between members of a group. Externalization is a tacit-to-explicit flow, as individual knowledge is articulated in explicit form, for instance. This is similar in many respects to how the Explicit Path from above begins, where knowledge rises up from the tacit plane as it is made explicit.

Combination is an explicit-to-explicit flow, as one individual's explicit knowledge is combined with 
others', for instance. This resembles in many respects how knowledge flows across the reach dimension in the Explicit Path from above. Internalization is an explicit-to-tacit flow, as knowledge is learned and applied in the organization, for instance. This resembles in many respects how explicit knowledge is applied in the Explicit Path from above. This cycle continues as knowledge spirals out ever further in terms of organization reach through a process termed amplification.

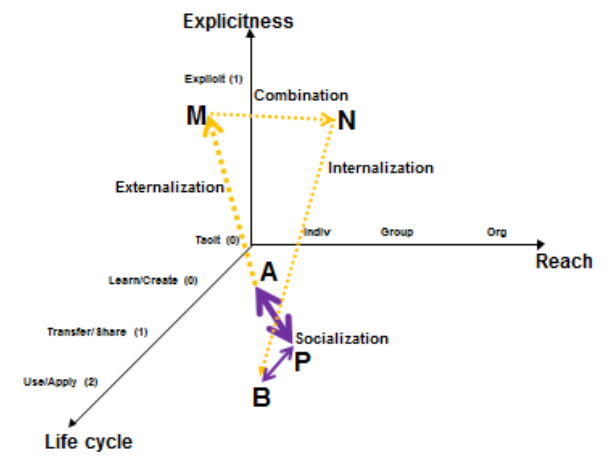

\section{Figure 6 Knowledge Spiral Representation}

As with the archetypical knowledge flow paths delineated above, we begin by representing the knowledge spiral via multidimensional space in Figure 6. To avoid cluttering the figure, we illustrate only one loop of the spiral.

Following the description above, we begin the knowledge spiral with socialization, which involves both direct experience and interpersonal interaction [15:19]. The experiential component is represented by a solid (purple) bidirectional arrow between Points $\mathrm{B}$ and $\mathrm{P}$. This represents people in a group working, learning and sharing experiences together. With the thin vector arrow, from KFT, we assume here that most people in the group are experienced and competent, enabling relatively quick application of their knowledge to perform organization work. With less experience and longer flow times, we would delineate such knowledge flow with a thicker arrow. The arrow is bidirectional to indicate knowledge flows for both work performance and experiential sharing.

The interactional component is represented by a solid (purple) bidirectional arrow between Points $\mathrm{P}$ and A. This represents people in a group interacting together with an individual at Point A. With the thick vector arrow, we assume here the individual to be comparatively new to the group and gaining tacit knowledge through socialization. From KFT, this process is likely to be comparatively slow, hence the relatively thick knowledge flow arrow. The arrow is bidirectional to indicate that the individual learns from the group and vice versa. Socialization involves only tacit knowledge, hence the flows are all within the tacit plane of the figure.

The spiral continues with externalization, which involves articulation of tacit knowledge into explicit form. We illustrate such articulation through a dotted (orange) unidirectional arrow between Points A and M. This represents an individual at Point A converting his or her tacit knowledge into explicit form (e.g., via written document). From KFT, the externalization process is likely to be relatively slow, hence the thick arrow, as considerable time and effort are required often to articulate one's knowledge explicitly.

Combination follows with another dotted (orange) unidirectional arrow, here between Points $\mathrm{M}$ and $\mathrm{N}$. This represents the combination of extant explicit knowledge of different people, shown in the figure as belonging to a group. A relatively thin arrow is used to delineate this combination flow, from KFT, as the process would likely occur comparatively quickly with respect to socialization and externalization, particularly because the extant knowledge has been articulated into explicit form already.

Finally, internalization completes the loop with a dotted (orange) unidirectional arrow between Points $\mathrm{N}$ and $\mathrm{B}$. This represents group learning through application of the knowledge combined from above, which we delineate with a relatively thin arrow, from KFT, to suggest that explicit knowledge flows comparatively quickly.

From here, the cycle can continue between individuals in the group, for instance, socializing, externalizing, combining and internalizing additional knowledge; or it can expand out to the organization level, for instance, as members of the group interact with people from different groups across the organization.

Clearly other interpretations of the knowledge spiral and their corresponding representations via multidimensional space are possible, but this illustrates at least one way in which spiraling knowledge can be delineated and visualized as with the knowledge flow archetypes above. Indeed, the knowledge spiral can be considered another archetype from the literature. We leave the identification and visualization of additional knowledge flow archetypes to future research.

Measurements corresponding to this knowledge spiral flow path are summarized in Table 8 and follow the same logic and procedure described for the archetypical paths above, reflecting, for instance, the same 100 chunks of knowledge and group size of 10 . 
Notice from our representation in multidimensional space how each flow shares much similarity with those used to delineate the archetypical knowledge flows above. For instance, the group knowledge application flow between Points $\mathrm{B}$ and $\mathrm{P}$ on the Spiral Path is similar to that between these same points on the Tacit Path: tacit knowledge is being applied by members of a group. Hence for consistency we show the same measurements in Table 8 as those reported in Table 6 (i.e., $E=0.0$; $\mathrm{KF}=1000 ; \mathrm{R}=10 ; \mathrm{FT}=0.1$ ).

Table 8 Spiral Path Measurement

\begin{tabular}{|l|c|c|r|r|r|r|}
\hline Flow & E & KF & \multicolumn{1}{c|}{ R } & \multicolumn{1}{c|}{ KW } & \multicolumn{1}{|c|}{ FT } & KP \\
\hline B-P & 0.0 & 1.00 & 10 & 10.0 & 0.1 & \\
\hline P-A & 0.0 & 1.00 & 10 & 10.0 & 55.0 & \\
\hline A-M & 0.5 & 0.55 & 1 & 0.55 & 15.3 & \\
\hline M-N & 1.0 & 0.10 & 10 & 1.00 & 0.1 & \\
\hline N-B & 1.0 & 0.10 & 10 & 1.00 & 1.0 & \\
\hline Sum & & & & 22.55 & 71.5 & 0.32 \\
\hline
\end{tabular}

Likewise, the sharing flow between Points $\mathrm{P}$ and A on the Spiral Path is similar to that between these same points on the Tacit Path: tacit knowledge is being shared between members of a group. Hence as above, we show the same measurements in Table 8 as those reported in Table 6 also.

Continuing, the individual articulation flow between Points $\mathrm{A}$ and $\mathrm{M}$ on the Spiral Path aligns well with that between these same points on the Explicit Path: tacit knowledge is being articulated in explicit form. Here we show the same measurements in Table 8 as those reported in Table 5. The same applies to explicit flows between Points $\mathrm{M}$ and $\mathrm{N}$ and between $\mathrm{N}$ and $\mathrm{B}$.

As summarized in Table 9, the spiral flow path accomplishes more knowledge work (22,550 P) than either of its explicit or tacit counterparts, but it also requires more time $(71,500 \mathrm{~s})$ to complete a loop. The knowledge power level (0.32) falls much closer to that of the tacit than the explicit flow archetype.

Table 9 Spiral Knowledge Flow Path Comparison

\begin{tabular}{|l|r|r|c|}
\hline Path & \multicolumn{1}{|c|}{ KW } & FT & KP \\
\hline Explicit & 2.55 & 16.1 & 0.16 \\
\hline Tacit & 20.00 & 55.2 & 0.36 \\
\hline Spiral & 22.55 & 71.5 & 0.32 \\
\hline
\end{tabular}

Thus, we are able to apply our knowledge visualization and measurement system to the very well-known knowledge spiral, and we show how to compare the spiral flow path with its explicit and tacit archetypical counterparts. This represents a substantial contribution of new knowledge and extension of the Spiral Model.

However, these dynamic knowledge measurements also lead us to question this longstanding, well-known theoretic model, which exhibits difficulties in such light. For instance, knowledge conversion is clearly key to the knowledge spiral, for such conversion underlies the flow and amplification of knowledge through an organization. Nonetheless, the Spiral Model is silent regarding the kind of knowledge energy loss that accompanies such conversion, particularly where tacit knowledge is articulated into explicit form (e.g., flow vector A-M).

Recall from our multidimensional visualization above, for instance, how explicit knowledge is characterized as flowing at lower energy levels than its tacit counterpart. Nonaka [15:16] quotes Polanyi [19] as saying that people know more than they can tell. Hence we understand that not all tacit knowledge can be articulated into explicit form.

Moreover, as noted above, explicit knowledge does not always enable action at the same performance level as the tacit from which it derives. Reading a book (i.e., explicit knowledge), for example, about how to fly an airplane is not the same as having spent time flying airplanes (i.e., tacit knowledge). When even an expert pilot is able to write down his or her knowledge, somethingsometimes extremely much - is lost generally, and the corresponding actions may not be performable at the same proficiency levels, say by a novice pilot. This represents knowledge flowing with less energy.

Further, we can quantify such energy loss by reviewing the dynamic knowledge measurements summarized in Table 8. Notice, for instance, that the K-Work (and K-Energy) accomplished through the first two flows (i.e., B-P, P-A) drops tenfold in the final two flows (i.e., M-N, N-B) after tacit knowledge has been articulated into explicit form. This is for the same 100 knowledge chunks, yet K-Energy loss reflects an order of magnitude.

Indeed, this is quite the opposite of knowledge becoming amplified through a spiral flow path, as theorized in the Spiral Model: with each conversion from tacit to explicit knowledge, the level of knowledge energy available to accomplish knowledge work decreases. Moreover, from this observation, the more often that knowledge cycles through a spiral flow path, the more that its energy level will decrease, and the less knowledge work will be accomplished in an organization.

Again, sensitivity analysis suggests that these results are highly robust (e.g., to slope, linearity, other factors). This suggests that it's time to 
reconsider the Spiral Model, along with its underlying assumptions and theoretic implications. This also represents a substantial, theoretic advance in terms of knowledge measurement. It remains for future research to address other theoretic models, and it calls for empiric research to measure the knowledge of operational organizations in the field. Knowledge measurement remains a nascent research endeavor. Even small, admittedly imprecise, analogic, theoretic steps such as ours can contribute much. We welcome others to contribute likewise.

\section{References}

[1] Ahn, J., \& Chang, S. (2004). Assessing the contribution of knowledge to business performance: The KP3 methodology. Decision Support Systems, 36(4), 403-416.

[2] Casakin, H. (2010). Visual analogy, visual displays, and the nature of design problems: The effect of expertise.

Environment and Planning B: Planning and Design, 37(1), 170-188.

[3] Cole, R. E. (1998). Introduction. California Management Review, 40(3), 15-21.

[4] Dierickx, I., \& Cool, K. (1989). Asset stock accumulation and sustainability of competitive advantage. Management Science, 35(12), 1504-1511.

[5] Drucker, P. F. (1995). Managing in a time of great change. New York: Truman Talley Books/Dutton.

[6] Gavetti, G., \& Rivkin, J. W. (2005). How strategists really think: Tapping the power of analogy. Harvard Business Review, 83(4), 54-63.

[7] Grant, R. M. (1996). Toward a knowledge based theory of the firm. Strategic Management Journal, 17, 109-122. Hayek, F.A. (1937). Economics and Knowledge.

Economica, 4(13), 33-54.

[8] Kolodner, J. L. (1994). Understanding creativity: A case-based approach. In: S. Wess S., K. Althoff, \& M. Richter (Eds.). Topics in Case-Based Reasoning. Berlin: Springer.

[9] Naim, M. M., Spiegler, V. M., Wikner, J., \& Towill, D. R. (2017). Identifying the causes of the bullwhip effect by exploiting control block diagram manipulation with analogical reasoning. European Journal of Operational Research, 263(1), 240-246.

[10] Nissen, M. E. (2002). An extended model of knowledge-flow dynamics. Communications of the Association for Information Systems, 8(18), 251-266.

[11] Nissen, M. E. (2006a). Dynamic Knowledge Patterns to Inform Design: A Field Study of Knowledge Stocks and
Flows in an Extreme Organization. Journal of Management Information Systems 22(3), 225-263.

[12] Nissen, M. E. (2006b). Harnessing knowledge dynamics: Principled organizational knowing and learning. Hershey, PA: IRM Press.

[13] Nissen, M. E. (2014). Harnessing dynamic knowledge principles for competitive advantage in the technologydriven world. Hershey, PA: IGI Global.

[14] Nissen, M. E. (2017). Working toward a system for measuring dynamic knowledge. International Journal of Knowledge Management, 13(3), 1-19.

[15] Nonaka, I. (1994). A dynamic theory of organizational knowledge creation. Organization Science, 5(1), 14-37.

[16] Peterson, M. (1997). The use of analogies in developing outer space law. International Organization, 51(2), 245-274.

[17] Phye, G. D. (1997). Handbook of Academic Learning: Construction of Knowledge. Burlington: Elsevier Science.

[18] Podolefsky, N. S., \& Finkelstein, N.D. (2007).

Salience of representations and analogies in Physics. AIP Conference Proceedings, 951(1), 164-167.

[19] Polanyi, M. (1967). The tacit dimension. Garden City, N.Y.: Anchor Books.

[20] Preiss, K. (1999). Modelling of Knowledge Flows and their Impact. Journal of Knowledge Management 3:1, pp. 36-46.

[21] Simon, H. A. (1996). The Sciences of the Artificial (3rd. ed.). Cambridge, MA: The MIT Press.

[22] Spender, J. C. (1996). Making knowledge the basis of a dynamic theory of the firm. Strategic Management Journal, 17, 45-62.

[23] Sternberg, R. J. (1977). Intelligence, information processing, and analogical reasoning: The componential analysis of human abilities. Hillsdale, N.J.: Lawrence Erlbaum Associates.

[24] Sternberg, R. J., \& Rifkin, B. (1979). The development of analogical reasoning processes. Journal of Experimental Child Psychology, 27(2), 195-232.

[25] Szulanski, G. (2000). The Process of Knowledge Transfer: A Diachronic Analysis of Stickiness. Organizational Behavior and Human Decision Processes 82:1, 9-27.

[26] Tsoukas, H. (1993). Analogical reasoning and knowledge generation in organization theory. Organization Studies, 14(3), 323-346. 